\title{
Cervical glandular atypia associated with squamous intraepithelial neoplasia: a premalignant lesion?
}

\author{
LJR BROWN, M WELLS \\ From the Department of Pathology, University of Leeds, Leeds
}

SUMMARY Recent studies have described premalignant changes in the endocervical epithelium, but morphological criteria for the diagnosis of cervical glandular atypia of lesser severity than adenocarcinoma in situ have not been established. Adenocarcinoma in situ is often associated with cervical intraepithelial neoplasia (CIN). The endocervical mucosa in 105 cases of CIN grade III was evaluated and compared with that of 100 controls. Sixteen cases of cervical glandular atypia and one case of adenocarcinoma in situ were identified, and it was possible to discriminate between these and a range of benign glandular lesions. Interestingly, the control series included two patients with cervical glandular atypia, one of whom on review had had a cone biopsy for CIN. The progression of cervical glandular atypia through adenocarcinoma in situ to invasive adenocarcinoma is known, but the natural history of cervical glandular atypia is as yet uncertain.

Adenocarcinoma in situ of the cervix was first described in 1953 in a patient who, coincidentally, had ectocervical carcinoma in situ. ${ }^{1}$ It is a lesion that is often overlooked, ${ }^{2-6}$ and which is strongly associated with squamous intraepithelial and invasive neoplasia. ${ }^{6-11}$ In exfoliative cytology abnormal columnar cells may accompany dyskaryotic squamous cells, providing evidence of adenocarcinoma in situ or invasive adenocarcinoma. ${ }^{268912}$ Comparison with the behaviour of premalignant epithelial lesions in other sites might presuppose that endocervical glands would also show a range of precancerous change. In the past such changes have been identified ${ }^{13}$ and an attempt at characterisation using computerised morphometry ${ }^{6}$ has been made. In this paper we attempted to establish the histological criteria for a range of possibly premalignant appearances that constitute cervical glandular atypia, and to indicate the incidence of their association with cervical intraepithelial neoplasia grade III (CIN III).

\section{Material and methods}

One hundred and five patients were selected, in whom a previous diagnosis of CIN III had been confirmed using standard histological criteria. ${ }^{14}$ To maximise the area of endocervical tissue available for review

Accepted for publication 16 September 1985 suitable cases were limited to hysterectomy and substantial cone biopsy specimens. One hundred hysterectomy specimens showing neither squamous intraepithelial nor invasive neoplasia were used as controls. The ages of the patients were recorded, and their menstrual state at the time of biopsy determined from the endometrium and clinical history.

The slides were examined on three separate occasions using a double headed Leitz Dialux microscope. Abnormalities of the endocervical glands were recorded in both the test and control groups. Thus we established architectural and cytological criteria for cervical glandular atypia, and benign lesions such as isthmic mucosa, endometriosis, microglandular hyperplasia, reserve cell hyperplasia, viral effect, tubal metaplasia, and mesonephric (Gartner's) duct remnants were excluded. Areas showing inflammation with reactive changes, which could be confused with atypia, were disregarded. ${ }^{15}$

\section{Results}

Tables 1, 2, and 3 show the incidence of the various glandular abnormalities, the age distribution, and the menstrual state at the time of biopsy of the patients.

The following benign conditions were identified and excluded from the cervical glandular atypia category:

Gartner's duct hyperplasia These mesonephric remnants appeared as small, regularly shaped, occasionally tightly packed acini lined by a single 
Table 1 Incidence of glandular abnormalities

\begin{tabular}{lll}
\hline & $\begin{array}{l}\text { Test }(\%) \\
(n=105)\end{array}$ & $\begin{array}{l}\text { Control } \\
(n=100)\end{array}$ \\
\hline Cervical glandular atypia & $16(15)$ & 2 \\
Adenocarcinoma in situ & $1(1)$ & 1 \\
Gartner's duct remnants & $12(11)$ & 4 \\
Microglandular hyperplasia & $28(27)$ & 8 \\
Reserve cell hyperplasia & $7(7)$ & 9 \\
Endometriosis & $4(4)$ & 3 \\
Multinucleate cells (? virus) & $3(3)$ & 2 \\
Tubal metaplasia & $4(4)$ & - \\
\hline
\end{tabular}

Figures in parentheses are numbers $\%$.

layer of pale staining cells with regular vesicular nuclei. Pink homogeneous secretion was often present.

Microglandular hyperplasia This consisted of collections of small banal glands occasionally forming polypoid projections into the endocervical canal. Accompanying inflammation and reserve cell hyperplasia were commonly seen.

Reserve cell hyperplasia A layer of glandular epithelium was seen overlying proliferating basal reserve cells, which, in some cases, comprised several layers. Although there was often increased staining, nuclear atypia was not seen.

Endometriosis Endometrial glands with stroma sometimes showing signs of haemorrhage were clearly recognised as being endometriotic in nature.

Multinucleate cells In five cases bizarre multinucleate cells were present in acini that showed no evidence of cervical glandular atypia (Fig. 1). There was no particular association with CIN (controls $2 \%$, CIN 3\%). In all these cases there was associated koilocytosis of the squamous epithelium, indicating human papilloma virus infection.

Tubal metaplasia Glands were lined by columnar ciliated, secretory, and small dark intercalated cells. The epithelium was similar to that of the fallopian tube.

Isthmic mucosa Lower segment glands were often seen in the upper endocervical canal, distinguished from cervical glandular atypia by their resemblance to endometrial glands, regular nuclear morphology, open chromatin, and low nuclear: cytoplasmic ratio.
Table 2 Age distribution in test patients with cervical glandular atypia, CIN, or both, or adenocarcinoma in situ and controls

\begin{tabular}{lll}
\hline Neoplasia & Mean age (years) & Range (years) \\
\hline Cervical glandular atypia & & \\
$\quad$ and CIN & $36 \cdot 9$ & $27-50$ \\
CIN & $34 \cdot 2$ & $22-55$ \\
Cervical glandular atypia & $36 \cdot 0$ & 33,39 \\
$\quad$ in control series & & \\
$\begin{array}{l}\text { Adenocarcinoma in situ } \\
\quad \text { one case) }\end{array}$ & 33 & $23-72$ \\
$\begin{array}{l}\text { Controls } \\
\text { Patronts }\end{array}$ & $42 \cdot 2$ & \\
\hline
\end{tabular}

Patients with cervical glandular atypia were not significantly ( $p=0.863 ;$ Mann-Whitney) older than those with CIN

Mitoses were often prominent.

Cervical glandular atypia Sixteen examples of cervical glandular atypia and one of adenocarcinoma in situ were seen in association with CIN.

High grade cervical glandular atypia was

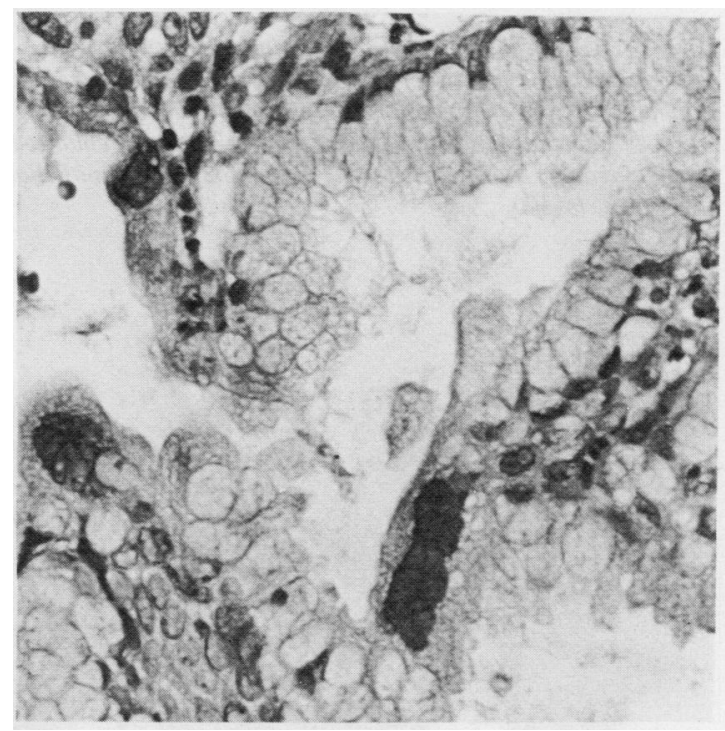

Fig. 1 Three multinucleate giant cells within endocervical gland. Surrounding epithelium appears normal with small basally located nuclei, small nuclear cytoplasmic ratio, and good mucin production. $\times 150$.

Table 3 Menstrual state at time of biopsy

\begin{tabular}{lllll}
\hline & CIN & $\begin{array}{l}\text { Cervical glandular } \\
\text { atypia and CIN }\end{array}$ & Control & $\begin{array}{l}\text { Cervical glandular } \\
\text { atypia in controls }\end{array}$ \\
\hline $\begin{array}{llll}\text { Secretory } \\
\text { Proliferative }\end{array}$ & 32 & 6 & 42 & 2 \\
$\begin{array}{l}\text { Menstrual } \\
\text { Inactive }\end{array}$ & 34 & 8 & 37 & \\
$\begin{array}{l}\text { Oral contraceptive } \\
\text { Postpartum }\end{array}$ & 2 & 1 & 5 & \\
$\begin{array}{l}\text { Hyperplastic } \\
\text { Pregnant }\end{array}$ & 1 & 1 & 2 \\
\hline
\end{tabular}






Fig. 2 Area of high grade cervical glandular atypia within gland containing foci of almost normal epithelium. In cervical glandular atypia epithelium is thrown up into intraluminal tufts with nuclei, which are densely hyperchromatic, enlarged, and elongated. Nuclei are present throughout entire epithelial thickness. $\times 100$.

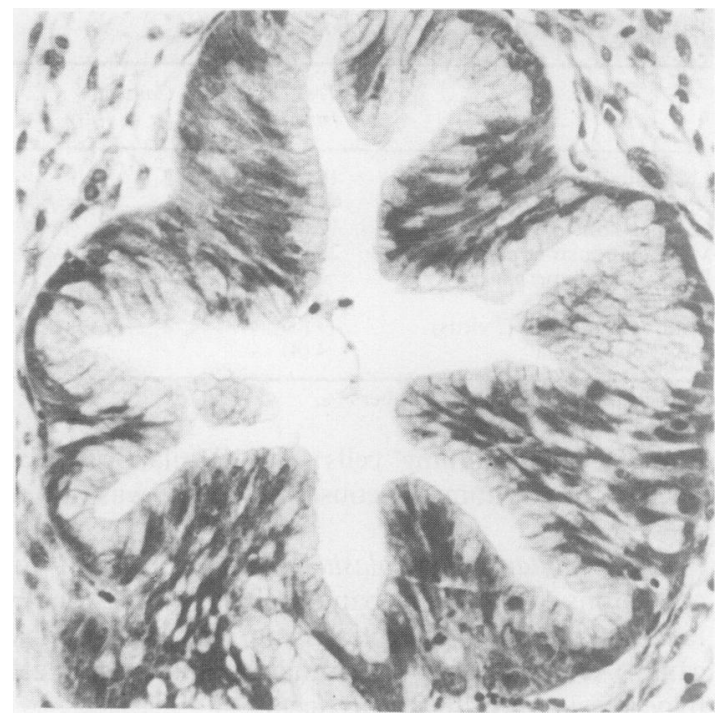

Fig. 3 Gland exhibiting high grade cervical glandular atypia with intraluminal tufting. For the most part nuclei occupy more than two thirds of the height of the epithelium. Mucin production is reduced and there are occasional subnuclear vacuoles. $\times 64$.

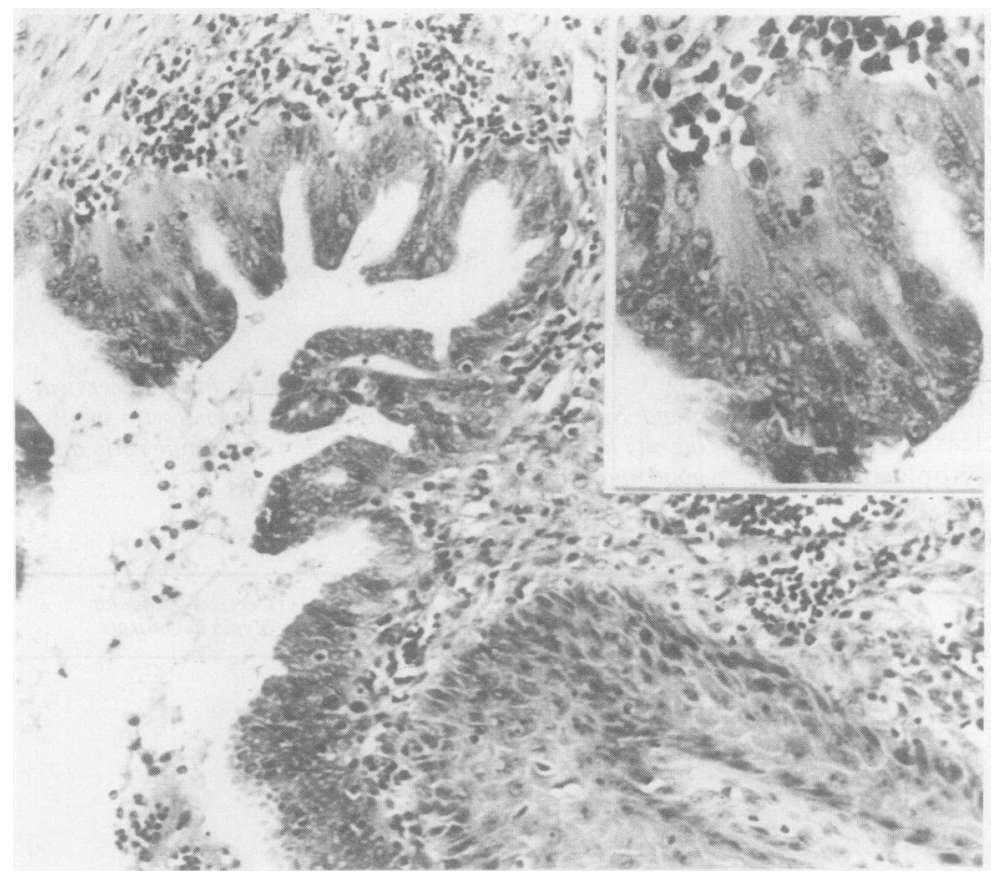

Fig. 4 High grade cervical glandular atypia underlying area of CIN. Many intraluminal papillary projections have no stromal core. Nuclear enlargement, pleomorphism, and stratification are pronounced. Note variation in chromatin pattern and occasional vesicular nucleus. Inset shows detail of epithelium at higher power. Very little mucin is apparent. $\times 40$ (inset $\times 250$ ). 


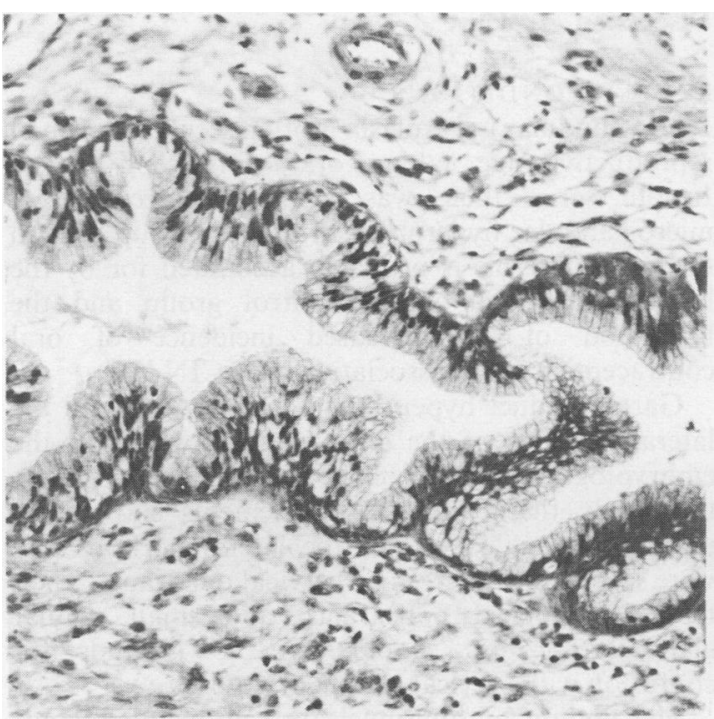

Fig. 5 This gland shows low grade cervical glandular atypia and exhibits intraluminal tufting and some irregularity of shape. Nuclei occupy less than two thirds of the height of the epithelium; there is minor variation of nuclear shape, size, and staining. Occasional subnuclear mucin vacuoles can be seen. $\times 40$.

characterised by nuclear pleomorphism, hyperchromatic occasionally vesicular nuclei, an increased nuclear:cytoplasmic ratio, nuclear stratification greater than two thirds of the epithelial height, and elongated enlarged nuclei (Fig. 2). Mitoses were rarely seen (less than one mitotic figure per glandular profile), and the chromatin was often arranged in irregular dense clumps. Most affected glands had an abnormal profile with irregular branching and budding. The atypical epithelium was irregularly heaped up to form intraluminal tufts and occasional low papillary projections lacking stromal cores (Figs. 3 and 4). One or two otherwise normal glands contained foci of high grade cervical glandular atypia with an abrupt transition from apparently normal columnar epithelium to severely atypical epithelium (Fig. 2).

Low grade cervical glandular atypia (Fig. 5) exhibited a spectrum of appearances ranging from minimally deviated glandular epithelium to changes similar to but less severe than high grade cervical glandular atypia. In its most pronounced forms low grade cervical glandular atypia showed luminal tufting, but all papillary projections apparently possessed stromal cores. Glandular profiles were abnormal and similar to those seen in high grade cervical glandular atypia (Fig. 6). The nuclei were enlarged, hyperchromatic, and elongated but

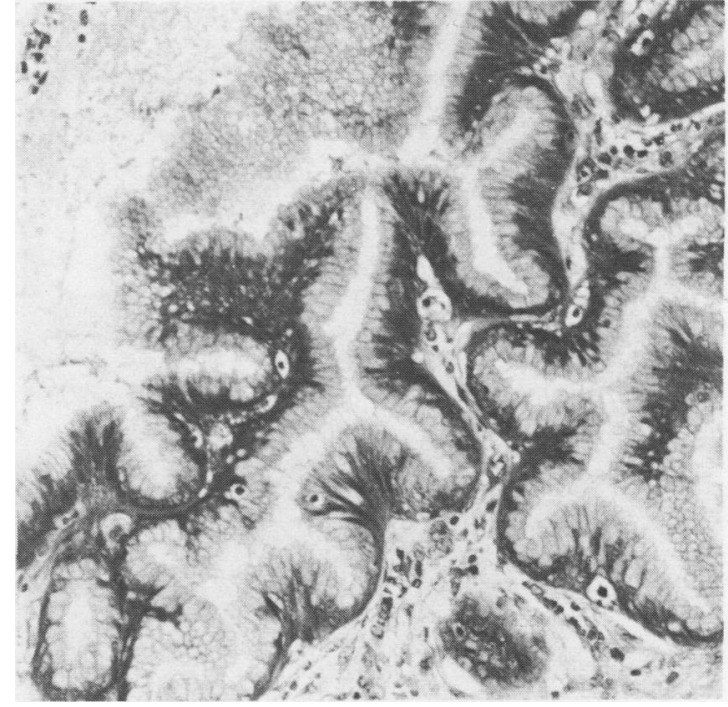

Fig. 6 Complex infolding, branching, and budding seen in cervical glandular atypia can be seen clearly in this field. $\times$ 40.

stratification was limited to the basal two thirds of the epithelium. Mitoses were not seen and vesicular nuclei were not as common as in high grade cervical glandular atypia. In its mildest form low grade cervical glandular atypia exhibited minor variations in nuclear size, shape, and staining. Irregularity of nuclear position was observed, but all nuclei were generally limited to the basal half of the epithelium. Glandular morphology was sometimes severely irregular but usually approximated to normal.

The whole spectrum of changes in cervical glandular atypia showed abnormalities of mucin production with absolute or partial mucin depletion. Subnuclear vacuolation was a common finding, but neither intestinal metaplasia nor goblet cells were seen. ${ }^{16}$ The salient morphological features of cervical glandular atypia were: nuclear stratification; increased nuclear size; increased nuclear to cytoplasmic ratio; increased nuclear staining; nuclear pleomorphism or abnormal chromatin pattern; increased mitotic activity; subnuclear vacuolation; partial or total mucin depletion; abnormal gland architecture; intraluminal tufting or papillary projections.

Of sixteen examples of cervical glandular atypia, seven were classified as high grade; the remainder as low grade. Irrespective of grade, cervical glandular atypia was located randomly in the glandular 


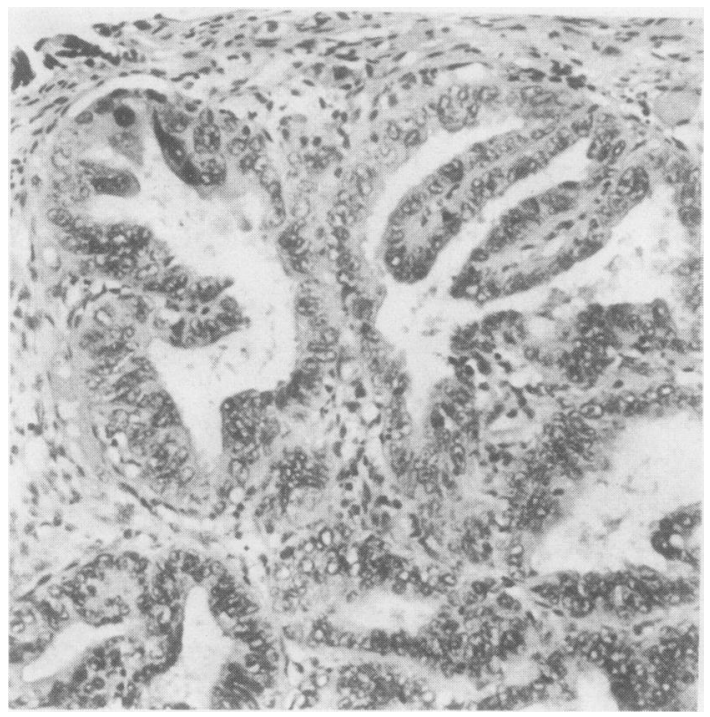

Fig. 7 Adenocarcinoma in situ showing high mitotic rate, glandular crowding, back to backing, and intraluminal papillary projections. $\times 40$.

endocervix and occasionally high in the endocervical canal. The amount of atypical epithelium varied from a small focus within an otherwise normal gland to many glands lined wholly by atypical epithelium. Atypical glands were never seen deeper than the level of normal endocervical glands. One of the two patients with cervical glandular atypia in the control series was subsequently discovered to have had a previous cone biopsy for CIN grade II.

The morphology of adenocarcinoma in situ has been amply described. ${ }^{1-5101217}$ In contrast to high grade cervical glandular atypia, the single case of adenocarcinoma in situ (Fig. 7) showed a more bizarre appearance with a greater degree of aberrant glandular morphology. In addition to stratification, the nuclei of adenocarcinoma in situ were often grossly enlarged to two or three times the normal size and occasionally seemed to fill the whole cell. Numerous mitoses (up to five) could be seen within the same gland and there were occasional tripolar forms. Generally, the glands were smaller than those seen in cervical glandular atypia and showed areas of crowding, back to backing, and cribriform patterns not seen in cervical glandular atypia. Overall, cellular atypia was more pronounced in adenocarcinoma in situ than in cervical glandular atypia. Glands showing changes identical to those seen in cervical glandular atypia were associated with adenocarcinoma in situ and apparently formed transition zones between adenocarcinoma in situ and normal epithelium.

\section{Discussion}

\section{BENIGN CONDITIONS}

Microglandular hyperplasia is often seen in women of reproductive age taking oral contraceptives. ${ }^{1819}$ In our series there was a higher percentage of microglandular hyperplasia in the test group than in the controls. This is probably accounted for by the higher mean age of the control group and the likelihood of an increased incidence of oral contraceptive use in association with CIN III.

Gartner's duct hyperplasia is said to occur in the lateral portions of the cervix corresponding to the embryological position of the mesonephric duct. ${ }^{5}$ In this study these hyperplastic elements showed no particular predilection for the lateral portions and were seen in blocks from all cervical quadrants. Their bland cytological appearance and small regular glandular profiles should give no grounds for confusion with cervical glandular atypia.

The finding of multinucleate cells within glands showed an interesting association with ectocervical viral change and bears an obvious similarity to viral lesions in other sites. In common with other workers ${ }^{20}$ we suggest that this appearance is probably a manifestation of viral infection of endocervical glandular cells.

\section{CERVICAL GLANDULAR ATYPIA}

Dysplastic changes in the endocervical glands adjacent to squamous cervical intraepithelial neoplasia have been described, ${ }^{613}$ but to date there seems to be little information on the incidence or age range of such atypias. In addition to morphological changes, changes in typical mucin staining patterns similar to those seen in the transitional mucosa of the large bowel have recently been described and may indicate premalignant mucosa. ${ }^{21}$ Areas of intestinal metaplasia have recently been described ${ }^{16}$ in the uterine cervix and might provide a basis for neoplastic change.

Ratios of in situ adenocarcinoma to CIN III have been given as $1: 26^{2}$ and 1:239 18 ; we found a ratio of 1:104. Cervical glandular atypia of a lesser severity than adenocarcinoma in situ occurred in $15 \%$ of our patients, making a ratio of cervical glandular atypia to CIN III of about 1:7. This shows that cervical glandular atypia is 16 times more common than adenocarcinoma in situ, as might be expected of a precursor lesion, but that only a proportion of cases appear to progress.

Cervical glandular atypia occurs in a younger age group than adenocarcinoma in situ. In this series we found a mean age for cervical glandular atypia of 36.9 years (range 27-50). The age of the one patient with adenocarcinoma in situ was 33 , but others have found 
a mean age for adenocarcinoma in situ of 39 years (range 22-66) ${ }^{1213}$ and 38.4 years (range 26-58). ${ }^{4}$ Microinvasive adenocarcinoma has been described as occurring at a mean age of 44 years (range 29-57), ${ }^{13}$ and deeply invasive adenocarcinoma at $57^{12}$ and 49 years (range $34-63$ ).${ }^{13} \mathrm{~A}$ mean age of 36 years (range 33-38) has been given ${ }^{13}$ for endocervical dysplasia, but this was in a series of only three cases. It is thought that adenocarcinoma in situ takes at least three to seven years to progress to invasive malignancy. ${ }^{12}$ From these figures a possible progression from cervical glandular atypia to adenocarcinoma in situ could be expected in one and a half to three years and from cervical glandular atypia to invasive adenocarcinoma in 12-20 years. Interestingly, our study showed a trend for cervical glandular atypia to occur in an older age group than CIN III (36.9 years for cervical glandular atypia, 34.2 years for CIN III), although this was not statistically significant $(p=0.863)$, and that for the two incidental cases of cervical glandular atypia in the normal series the patients were also older (36 years) than the mean age for CIN III. The theory has been advanced $^{2418}$ that both squamous neoplasms and adenocarcinomas derive from reserve cells, possibly in response to viral infection, ${ }^{3}$ but that the glandular epithelium takes longer to develop its malignant potential. Our results showed a strong association of cervical glandular atypia with CIN (15\% compared to $2 \%$ in the controls), suggesting a common causative factor and indicate that on average cervical glandular atypia takes 2.7 years longer to develop than CIN III. Even in the control series one of the two cases of cervical glandular atypia had been associated with CIN. Nevertheless, in the five cases exhibiting glandular viral changes there were no signs of cervical glandular atypia, and although ectocervical viral changes were often present in association with cervical glandular atypia, in none of these cases were multinucleate cells seen in the glandular epithelium.

The menstrual state at the time of biopsy for every example of cervical glandular atypia was ascertained. Nearly twice as many cases were in the proliferative (10) as in the secretory phase (six). The numbers were small, however, and there were also single cases of cervical glandular atypia associated with the use of oral contraceptives and the menstrual and hyperplastic endometrium. These results indicated that cervical glandular atypia is not related to the phase of the menstrual cycle and should refute any suggestions that cervical glandular atypia is a spurious appearance resulting from endocervical cyclical changes.

Classification of premalignant epithelium of the colon into mild, moderate, and severe grades is unreliable even when undertaken by informed observers. ${ }^{22}$ Agreement between observers in grading is improved when the presence or absence of severe dysplasia is sought specifically ${ }^{22}$ and for this reason we used only two grades of cervical glandular atypia. When the behaviour of cervical glandular atypia has been fully elucidated follow up or therapeutic regimens may come to be based on a particular grade of cervical glandular atypia, but for the present it seems prudent to regard the whole spectrum of changes in cervical glandular atypia as one lesion. Furthermore, of course, the distinction between high grade cervical glandular atypia and adenocarcinoma in situ may well prove, biologically and therapeutically, to be an artificial one.

To conclude, in describing cervical glandular atypia we characterised a spectrum of atypical changes, including enlarged irregular glandular profiles with intraluminal tufts and papillae, which probably reflect rapid cellular proliferation. Cervical glandular atypia is unrelated to the menstrual cycle, is similar although less severe than adenocarcinoma in situ, occurs in areas bordering adenocarcinoma in situ, and occurs at an earlier age than adenocarcinoma in situ. We believe that this is good evidence of a neoplastic progression from cervical glandular atypia to adenocarcinoma in situ, and that in view of this the presence of cervical glandular atypia should be commented on in standard reports and should at least initiate cytological follow up with particular attention to atypical columnar cells. Although the presence of atypical columnar cells accompanying dyskaryotic squamous cells in a smear has often been quoted 268912 as being indicative of the presence of dysplastic glandular epithelium, a recent study showed that this criterion is not infallible and that it is often difficult to distinguish between abnormal squamous and dysplastic glandular cells (unpublished observations, JD Hemming, JM Light). In addition, we also showed that cervical glandular atypia is multifocal and often located high in the endocervical canal. A similar distribution is seen with adenocarcinoma in situ, and numerous workers have noted that such lesions can be missed by colposcopic punch, or even cone biopsy. ${ }^{3468917}$

\section{References}

${ }^{1}$ Freidell GH, McKay DG. Adenocarcinoma in situ of the endocervix. Cancer 1953;6:887-97.

${ }^{2}$ Boon ME, Kirk RS, Rietveld-Scheffers PEM. The morphogenesis of adenocarcinoma of the cervix-a complex pathological entity. Histopathology 1981;5:565-77.

${ }^{3}$ Buscema J, Woodruff JD. The significance of neoplastic atypicalities in endocervical epithelium. Gynecol Oncol 1984;17:356-62.

${ }^{4}$ Qizilbash A. In situ and microinvasive adenocarcinoma of the uterine cervix. Am J Clin Pathol 1975;64:155-70.

${ }^{5}$ Ostör AG, Pagano R, Davoren RA, Fortune DW, Chanen W, 
Rome R. Adenocarcinoma in situ of the cervix. Int $J$ Gynecol Pathol 1984;3:179-90.

${ }^{6}$ Van Roon E, Boon ME, Kurver PJH, Baak JPA. The association between precancerous columnar and squamous lesions of the cervix: a morphometric study. Histopathology 1983;7:887-96.

${ }^{7}$ Maier RC, Norris HJ. Coexistence of cervical intraepithelial neoplasia with primary adenocarcinoma of the endocervix. Obstet Gynecol 1980;56:361-4.

${ }^{8}$ Wolk BM, Kime W, Albites V. Simultaneous in situ squamous cell carcinoma and microinvasive adenocarcinoma of the cervix. In J Gynaecol Obstet 1981:19:69-72.

${ }^{9}$ Rosenthal DL, McLatchie C, Stern E, White BS, Castleman KR. Endocervical columnar cell atypia coincident with cervical neoplasia characterised by digital image analysis. Acta Cytol 1982;26:115-20.

${ }^{10}$ Gloor E, Ruzicka J. Morphology of adenocarcinoma-in-situ of the uterine cervix: a study of 14 cases. Cancer 1982;49:294-302.

"Choo YC, Naylor B. Co-existent squamous cell carcinoma and adenocarcinoma of the uterine cervix. Gynecol Oncol 1984;17:168-74.

12 Boon ME, Baak JPA, Kurver PJH, Overdiep SH, Verdonk GW. Adenocarcinoma in situ of the cervix: and underdiagnosed condition. Cancer 1981;48:768-73.

${ }^{13}$ Bousfield L, Pacey F, Young Q, Krumins I, Osborn R. Expanded cytological criteria for the diagnosis of adenocarcinoma in situ of the cervix and related lesions. Acta Cytol 1980;24:283-96.

${ }^{14}$ Buckley CH, Butler EB, Fox H. Cervical intraepithelial neoplasia.
J Clin Pathol 1982;35:1-13.

${ }^{15}$ Shingleton HM, Gore H, Bradley DH, Soong SJ. Adenocarcinoma of the cervix. I. Clinical evaluation and pathologic features. Am J Obstet Gynecol 1981;139:799-814.

${ }^{16}$ Trowell JE. Intestinal metaplasia with argentaffin cells in the uterine cervix. Histopathology 1985;9:551-9.

${ }^{17}$ Christopherson WM, Nealon N, Gray CA. Non invasive precursor lesions of adenocarcinoma and mixed adenosquamous carcinoma of the cervix uteri. Cancer 1979;44:975-83.

${ }^{18}$ Candy J, Abell MR. Progestogen-induced adenomatous hyperplasia of the uterine cervix. JAMA 1968;203:85-8.

${ }^{19}$ Nichols TM, Fidler HK. Microglandular hyperplasia in cervical cone biopsies taken for suspicious and positive cytology. Am J Clin Pathol 1971;56:424-9.

${ }^{20}$ Fox H, Buckley CH. Atlas of gynaecological pathology. Current histopathology. Vol 5, Austin Gresham G, ed. Lancaster: MTP Press, 1983:39-40.

${ }^{21}$ Wakefield E, Wells M. Histochemical study of endocervical glycoproteins throughout the normal menstrual cycle and adjacent to cervical intraepithelial neoplasia. International Journal of Gynecology Pathology 1985;4:230-9.

22 Brown LJR, Smeeton NC, Dixon MF. Assessment of dysplasia in colorectal adenomas: an observer variation and morphometric study. J Clin Pathol 1985;38:174-9.

Requests for reprints to: Dr M Wells, Department of Pathology, University of Leeds, Leeds LS2 9JT, England. 